\title{
Sample Chambers with Mother-Daughter Mode
}

\author{
P. A. Wilk, C. M. McGrath, K. E. Gregorich, D. C. Hoffman
}

\section{Introduction}

A set of eight stand-alone sample chambers with a common interface were constructed at LBNL for improved detection of alpha and fission decay chains over currently used designs. The stainless steel chambers (see Figure 1 for a schematic and Figure 2 for a photograph of a completed chamber) were constructed to allow for low background detection of a daughter event by removal of the sample following the detection of a parent event. This mother-daughter mode of operation has been utilized successfully with our Merry-go-Round (MG) detection system [Gregorich 1994].

\section{Description}

\section{Sample Chambers}

Each chamber contains a pair of detectors that sandwich the sample planchet. The detectors are mounted as close as possible to the sample to maximize geometric efficiency. The extent that this is possible is limited by the space required to allow for unhindered movement of the planchet. To reduce the total volume of the chambers, side mounted Ortec Ultra Alpha (Tu-021-450-100) detectors were used. The reported resolution for these detectors is $21 \mathrm{keV}$. They are biased at 50 volts positive. Flexible cabling connects the detectors to a 
vacuum-tight pass-through LEMO connector accessible from the back of the sample chamber. For daughter mode operation, the activity must be deposited on a film thin enough for alpha particles to penetrate easily. Thin polypropylene $\left(\sim 30-40 \mu \mathrm{g} / \mathrm{cm}^{-1}\right)$ has been used in the past, but if the sample needs to be evaporated under heat, a coated Mylar film is expected to work.

\section{Vacuum System}

All the sample chambers share a common vacuum source. A microcomputer controlled solenoid-actuated valve system opens each chamber individually to the vacuum. The valves were manufactured by Mead Fluid Dynamics. The pressure in each chamber is monitored individually with an electronic switch that provides an indication of the pressure. The switch is connected to a microcomputer, which can start and stop data acquisition depending on the pressure in the chamber. All fittings on the chambers are by Swagelok ${ }^{\mathrm{TM}}$.

\section{Piston Assembly}

The sample planchet is inserted and removed from its position between the detector pair by a compressed air driven piston. The compressed air can either be provided from a compressed gas cylinder, or if available, a house compressed air supply. The piston tube assembly was purchased from Bimba Manufacturing Company (stainless, BF-011 5-DB EE5) and it was affixed to the sample chambers with a custom designed attachment with a Wilson seal to preserve vacuum during actuation. The piston function is controlled by the microcomputer 
via a solenoid valve (Mead Fluid Dynamics, Nova I, N2-SE) that connects the chamber either to the pressurized air or atmosphere.

Actuation and Monitor Interface

All operation of the sample chamber system is controlled by a microcomputer running LabView software. The majority of the computer interface hardware is from National Instruments, and where appropriate their catalog number is given. All input/output operations are through optically isolated solid-state relays (SSRODC-5 \& SSR-IDC) plugged into a 24-channel backplane (24-backplane). With a flat cable adapter (SC-2054 \& NB5), this backplane is plugged into a 96-channel Digital Input-Output (PCI-DIO-96) interface board that is installed in the microcomputer. All the solenoid valves operate on $12 \mathrm{~V} \mathrm{DC}$ power provided by two, 6.8 Amp regulated power supplies (SLS-12-068) from SOLA. Eight of the relays operate the valves that control the vacuum that is applied to the chambers; eight of the relays operate the valves that operate the pistons, and the final eight relays are used to switch the data stream to the acquisition system off and on.

\section{Switch Register}

A panel of eight, single throw switches controls the vacuum sub-system. This allows for individual opening of each chamber to vacuum and then starting data acquisition from this chamber. This could have been accomplished via virtual switches on the LabView ${ }^{\mathrm{TM}}$ control panel, but it was felt that physical switches provide a more intuitive interface that could be operated during an experiment easily and with positive tactile feedback. Individual transitor-transitor logic (TTL) 
signals for the switch contact are generated from the $5 \mathrm{~V} D C$ power output from the relay backplane, which is connected to eight of the channels of the digital interface, and a large $(3.3 \mathrm{k} \Omega$ ) pull-up resistor with switch to ground [Horowitz 1989].

Data Acquisition

The data acquisition system operates independently of the detector operation.

This allows for the use of whatever acquisition system is currently being used at the experimental facility from which the data are being collected. As it was intended to be first used in Cave 0 of the 88-Inch Cyclotron, the acquisition system would then be the GOOSY (GSI Online-Offline SYstem) software package developed at GSI [Essel 1987] on a Digital Equipment VAX workstation running the VMS operating system. The interface between the crate and the workstation is the GSI crate controller, the CVC. The CVC is a CAMAC to VSB Computer with SCSI, Ethernet, VSB, and CAMAC interfaces.

\section{Bibliography}

[Essel 1987] H. G. Essel, H. Grein, T. Kroll, W. Kynast, M. Richter, H. Sohlbach, W. Spreng, K. Winkelmann, W. F. J. Müller, IEEE Transactions on Nuclear Science, NS-34, 907 (1987).

[Gregorich 1990] K. E. Gregorich, M. R. Lane, M. F. Mohar, D. M. Lee, C. D. Kacher, E. R. Sylwester, D. C. Hoffman, Physical Review Letters, 72, 1423 (1994).

[Horowitz 1989] Paul Horowitz and Winfield Hill, The Art of Electronics, $2^{\text {nd }}$ Edition, pg. 386 (Cambridge University Press, 1989).

[Wilk 2001] P. A. Wilk, Ph.D. Thesis: Properties of Group Five and Group Seven Transactinium Elements, LBNL-47475, pg. 30 (2001). 
Figure 1. Sample Chamber CAD Drawing

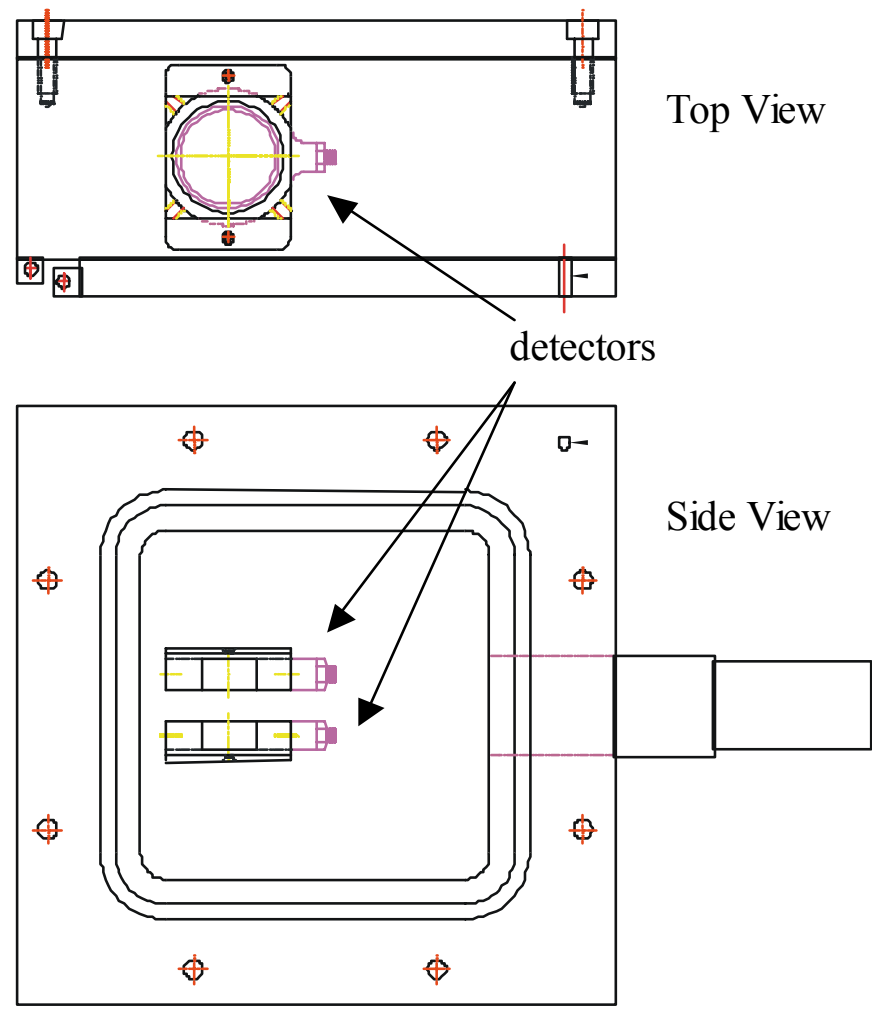

Drawing by C. M. McGrath

Figure 2. Sample Chamber Assembly without Detectors

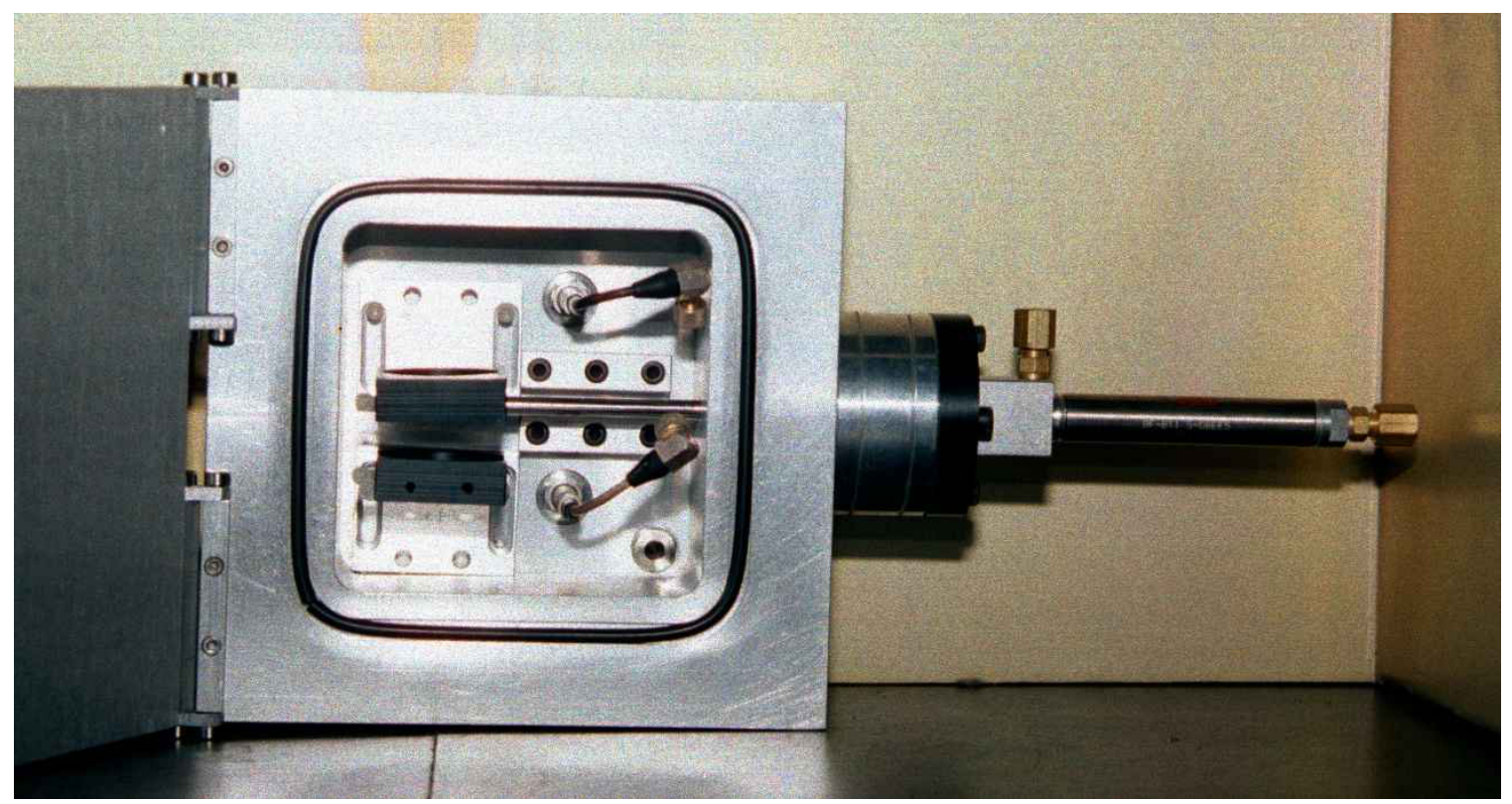

Photo by P. A. Wilk 